\title{
SIMPLIFIED CONTROLLER DESIGN FOR OUTPUT PERFORMANCE UNDER COMMON INPUT LIMITATIONS WITH GENERALIZED INTEGRAL ANTI-WINDUP FOR A CLASS OF PROCESSES
}

\author{
Taworn Benjanarasuth \\ Department of Instrumentation and Control Engineering, Faculty of Engineering, \\ King Mongkut's Institute of Technology Ladkrabang, Bangkok, Thailand, \\ Tel: +6623298353, e-mail: taworn.be@kmitl.ac.th
}

Received Date: October 15, 2019; Revised Date: January 18, 2020; Acceptance Date: January 28, 2020

\begin{abstract}
One of basic key tasks of a control system design is to achieve the desired output responses both in transient and steady states. Besides, the common input limitations, such as saturation and slew rate or at least avoiding a sudden jump in the command signal, must be considered in practice. However, popular controllers such as PI and PID cause sudden changes or even impulsive surges in the command signal under external excitations by a step reference input and/or step input/output disturbances. In this paper, a simplified controller design with its preferred structure models to meet the mentioned requirements is presented for a class of minimum-phase stable linear timeinvariant single-input single-output processes with proper real rational transfer function. The structure of such controller is mathematically investigated and the result is that the controller must be strictly proper and containing an integral factor. The design procedure is simple and straightforward based on reference model matching and model cancellation with only two required conditions on the desired closed-loop transfer function which are its relative degree comparing to the processes to be controlled and the equality of the lower order coefficient(s) in its numerator and denominator polynomials. A generalized integral anti-windup structure, based on back calculation method and PI/PID anti-windup scheme, to lessen the saturation effect on the integral action of the proposed controller is additionally introduced by rearranging the controller in a parallel form with one separated integral control action portion. Numerical examples are investigated to demonstrate the design procedure and verify the success of the proposed controller to the required objectives.
\end{abstract}

Keywords: Generalized integral anti-windup, Input limitations, Integral control, Model matching

\section{Introduction}

Designing a control system to meet desired response requirements is one of a fundamental concept in control engineering studies. Some well-known methods, such as pole placement techniques and reference model matching, have well been established and developed widely in much more sophisticated conditions. The idea of matching the closed-loop model of a simple negative unity feedback control with one adjustable controller to the target model algebraically has long been investigated [1]. With several physical constraints, the selection of such reference model might become quite difficult [2]. The well-known Youla parametrization (or more precisely Youla-Kučera parametrization) provides a formula describing all stabilizing controllers for a specified process model [3], [4] and can be adjusted if additional desired requirements are imposed. In a modern control paradigm, the idea of exact model matching based on by state feedback was also introduced firstly in [5]. Numerous works have extended and applied these fundamental ideas until nowadays such as [6], [7], [8]. However, designing procedures have become more and more sophisticated 
for general users. On the other hand, the old but still the most well-known PI/PID control [9] has widely been in use, such as [10], [11], despite the availability of all above mentioned control schemes and their subsequences due to the simplicity of PI/PID control for practitioners. Even under saturation constraints, the simple extended work of PI/PID anti-windup technique, later known as back calculation, was introduced firstly for digital implementation [12] to handle the windup due to the integral control action of a PI or PID controller. In fact, the integral windup is just a special case of a more general problem that all controllers including relatively slow or unstable modes would face under the input nonlinearity limitations [13]. Several schemes both for analog and digital domains have been studied to handle the saturation, for example the observer approach which must be considered via the modern control paradigm [14]. Some recent examples on extending and applying the anti-windup scheme ideas include [15], [16], [17].

In this paper, the simple idea in designing a controller for a class of minimum-phase stable linear time-invariant (LTI) single-input single-output (SISO) processes with proper real rational transfer function models. Although, this class of processes seems to be limited but many practical processes fall into the category including, but not limited to, several renewable energy processes such as wind turbine generators, diesel generator systems, fuel cell generators, aqua electrolyzer systems, and battery energy storage systems [18]. The proposed scheme is proven to result in the desired responses based on the reference model provided. The minimal conditions of the reference model are given so that the resulting controller is assured to eliminate the steady-state error due to step reference input excitation as well as step input and output disturbances excitations while avoiding sudden jumps or surges in the corresponding command signal. By expanding the back calculation method and well-known PI/PID antiwindup scheme, a generalized integral anti-windup is also presented to alleviate the saturation effect on the integral action of the proposed controller.

\section{Proposed Controller Structure and Design}

Consider the simple unity negative feedback control of an LTI SISO system in Figure 1 with process and controller transfer functions $P(s)$ and $C(s)$ respectively.

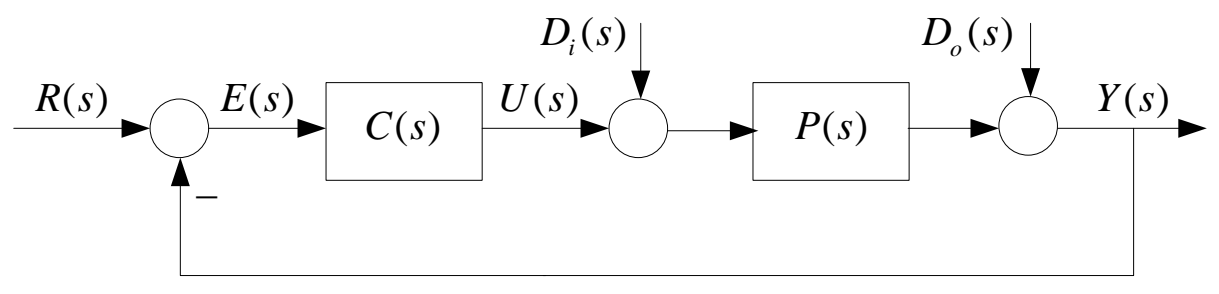

Figure 1. Control system under consideration

The process transfer function $P(s)$ and a controller transfer function $C(s)$ are assumed to be real and rational presented in (1) and (2) respectively.

$$
\begin{aligned}
& P(s)=\frac{N_{P}(s)}{D_{P}(s)}=\frac{b p_{m p} s^{m p}+b p_{m p-1} s^{m p-1}+\ldots+b p_{1} s+b p_{0}}{s^{n p}+a p_{n p-1} s^{n p-1}+\ldots+a p_{1} s+a p_{0}} \\
& C(s)=\frac{N_{C}(s)}{D_{C}(s)}=\frac{b c_{m c} s^{m c}+b c_{m c-1} s^{m c-1}+\ldots+b c_{1} s+b c_{0}}{s^{n c}+a c_{n c-1} s^{n c-1}+\ldots+a c_{1} s+a c_{0}}
\end{aligned}
$$


The nine transfer functions from the reference input $R(s)$, input disturbance $D_{i}(s)$ and output disturbance $D_{o}(s)$ to the error signal $E(s)$, command signal $U(s)$ and output $Y(s)$ can be computed as in (3).

$$
\begin{aligned}
{\left[\begin{array}{c}
E(s) \\
U(s) \\
Y(s)
\end{array}\right] } & =\left[\begin{array}{lll}
G_{11}(s) & G_{12}(s) & G_{13}(s) \\
G_{21}(s) & G_{22}(s) & G_{23}(s) \\
G_{31}(s) & G_{31}(s) & G_{33}(s)
\end{array}\right]\left[\begin{array}{c}
R(s) \\
D_{i}(s) \\
D_{o}(s)
\end{array}\right] \\
& =\frac{1}{1+P(s) C(s)}\left[\begin{array}{ccc}
1 & -P(s) & -1 \\
C(s) & -P(s) C(s) & -C(s) \\
P(s) C(s) & P(s) & 1
\end{array}\right]\left[\begin{array}{c}
R(s) \\
D_{i}(s) \\
D_{o}(s)
\end{array}\right] \\
& =\frac{\left[\begin{array}{lll}
D_{P}(s) D_{C}(s) & -N_{P}(s) D_{C}(s) & -D_{P}(s) D_{C}(s) \\
D_{P}(s) N_{C}(s) & -N_{P}(s) N_{C}(s) & -D_{P}(s) N_{C}(s) \\
N_{P}(s) N_{C}(s) & N_{P}(s) D_{C}(s) & D_{P}(s) D_{C}(s)
\end{array}\right]}{D_{P}(s) D_{C}(s)+N_{P}(s) N_{C}(s)}\left[\begin{array}{c}
R(s) \\
D_{i}(s) \\
D_{o}(s)
\end{array}\right]
\end{aligned}
$$

In this paper, the process transfer function $P(s)$ to be controlled in (1) is additionally assumed to be minimum-phase without zero at the origin, proper ( $n p \geq m p)$, and with all Left-Half-Plane (LHP) pole(s) without or with an integral factor.

\section{Sudden Jump in Command Signal Avoidance Condition}

Proposition 1: A sudden jump in the command signal $u(t)=L^{-1}\{U(s)\}$ under a step reference input and/or step input/output disturbances excitations can be avoided if the controller $C(s)$ is strictly proper.

Proof: Assume that the controller $C(s)$ in (2) is strictly proper or $n c>m c$. Since the system is LTI, it is sufficient to show that $\lim _{t \rightarrow 0} u(t)$ is equal to 0 due to unit step reference input excitation, unit step input disturbance excitation and unit step output disturbance excitation separately all at time $t=0$.

From the initial value theorem of Laplace transform, the initial value of $u(t)$ is related to its Laplace transform $U(s)$ as in (4).

$$
\lim _{t \rightarrow 0} u(t)=\lim _{s \rightarrow \infty} s U(s)
$$

Thus for the unit step reference input excitation at time $t=0$ or $R(s)=L\{r(t)\}=1 / s$, unit step input disturbance excitation at time $t=0$ or $D_{i}(s)=L\left\{d_{i}(t)\right\}=1 / s$ and unit step output disturbance excitation at time $t=0$ or $D_{o}(s)=L\left\{d_{o}(t)\right\}=1 / s, \lim _{t \rightarrow 0} u(t)$ for each excitation can be derived from (3) respectively as follows:

For the unit step reference input excitation

$$
\lim _{t \rightarrow 0} u(t)=\lim _{s \rightarrow \infty} s G_{21}(s) R(s)=\lim _{s \rightarrow \infty} G_{21}(s)=\lim _{s \rightarrow \infty} \frac{D_{P}(s) N_{C}(s)}{D_{P}(s) D_{C}(s)+N_{P}(s) N_{C}(s)}
$$


The order of the numerator term, $m c+n p$, is strictly less than and the order of the denominator term, $\max (n p+n c, m p+m c)=n p+n c$, since $n c>m c$; therefore, $\lim _{t \rightarrow 0} u(t)=0$.

$\underline{\text { For the unit step input disturbance excitation }}$

$$
\lim _{t \rightarrow 0} u(t)=\lim _{s \rightarrow \infty} s G_{22}(s) D_{i}(s)=\lim _{s \rightarrow \infty} G_{22}(s)=\lim _{s \rightarrow \infty} \frac{-N_{P}(s) N_{C}(s)}{D_{P}(s) D_{C}(s)+N_{P}(s) N_{C}(s)}
$$

The order of the numerator term, $m p+m c$, is strictly less than the order of the denominator term, $\max (n p+n c, m p+m c)=n p+n c$, since $n p \geq m p$ and $n c>m c$; therefore, $\lim _{t \rightarrow 0} u(t)=0$.

For the unit step output disturbance excitation

$$
\lim _{t \rightarrow 0} u(t)=\lim _{s \rightarrow \infty} s G_{23}(s) D_{o}(s)=\lim _{s \rightarrow \infty} G_{23}(s)=\lim _{s \rightarrow \infty} \frac{-D_{P}(s) N_{C}(s)}{D_{P}(s) D_{C}(s)+N_{P}(s) N_{C}(s)}
$$

The order of the numerator term, $n p+m c$, is strictly less than and the order of the denominator term, $\max (n p+n c, m p+m c)=n p+n c$, since $n c>m c$; therefore, $\lim _{t \rightarrow 0} u(t)=0$.

\section{Zero Steady-State Error Condition for Step Signal Excitations}

Proposition 2: A class of strictly proper controllers $C(s)$ to ensure zero steady-state error under step reference input and/or step input/output disturbances has an integral factor or its transfer function in the form of $(8)$ where $C_{1}(s)$ is proper and has no zero at the origin.

$$
C(s)=\frac{1}{s} C_{1}(s)
$$

Proof: Assume that the controller $C(s)$ makes the control system internally stable. Since the term $C_{1}(s)$ of (8) is proper and has no zero at the origin, it can be expressed as in (9) where $M=m c, N=n c-1$ and $N \geq M$.

$$
C_{1}(s)=\frac{N_{C 1}(s)}{D_{C 1}(s)}=\frac{q_{M} s^{M}+q_{M-1} s^{M-1}+\ldots+q_{1} s+q_{0}}{s^{N}+r_{N-1} s^{N-1}+\ldots+r_{1} s+r_{0}}, \text { where } q_{0} \neq 0
$$

From the final value theorem of Laplace transform, the steady-state value of the error $e(t)$, namely $e_{s s}$, is related to its Laplace transform $E(s)$ as in (10) if the control system internally stable.

$$
e_{s s}=\lim _{t \rightarrow \infty} e(t)=\lim _{s \rightarrow 0} s E(s)
$$

Since the system is LTI, it is sufficient to show that $e_{s s}$ is equal to 0 due to unit step reference input excitation, unit step input disturbance excitation and unit step output 
disturbance excitation separately all at time $t=0$. From (3), $e_{s s}$ for each excitation can be derived respectively as follows.

For the unit step reference input excitation

$$
\begin{aligned}
e_{s s} & =\lim _{s \rightarrow 0} s E(s)=\lim _{s \rightarrow 0} s G_{11}(s) R(s)=\lim _{s \rightarrow 0} G_{11}(s) \\
& =\lim _{s \rightarrow 0} \frac{D_{P}(s) D_{C}(s)}{D_{P}(s) D_{C}(s)+N_{P}(s) N_{C}(s)}=\lim _{s \rightarrow 0} \frac{s D_{P}(s) D_{C 1}(s)}{s D_{P}(s) D_{C 1}(s)+N_{P}(s) N_{C 1}(s)}
\end{aligned}
$$

For the unit step input disturbance excitation

$$
\begin{aligned}
e_{s s} & =\lim _{s \rightarrow 0} s E(s)=\lim _{s \rightarrow 0} s G_{12}(s) D_{i}(s)=\lim _{s \rightarrow 0} G_{12}(s) \\
& =\lim _{s \rightarrow 0} \frac{-N_{P}(s) D_{C}(s)}{D_{P}(s) D_{C}(s)+N_{P}(s) N_{C}(s)}=\lim _{s \rightarrow 0} \frac{-s N_{P}(s) D_{C 1}(s)}{s D_{P}(s) D_{C 1}(s)+N_{P}(s) N_{C 1}(s)}
\end{aligned}
$$

$\underline{\text { For the unit step output disturbance excitation }}$

$$
\begin{aligned}
e_{s s} & =\lim _{s \rightarrow 0} s E(s)=\lim _{s \rightarrow 0} s G_{13}(s) D_{o}(s)=\lim _{s \rightarrow 0} G_{13}(s) \\
& =\lim _{s \rightarrow 0} \frac{-D_{P}(s) D_{C}(s)}{D_{P}(s) D_{C}(s)+N_{P}(s) N_{C}(s)}=\lim _{s \rightarrow 0} \frac{-s D_{P}(s) D_{C 1}(s)}{s D_{P}(s) D_{C 1}(s)+N_{P}(s) N_{C 1}(s)}
\end{aligned}
$$

In all cases, since the process $P(s)$ is assumed to have no zero at the origin or $N_{P}(0) \neq 0$ and since $C_{1}(s)$ do not contain zero at the origin or $N_{C 1}(0) \neq 0$, the controller $C(s)$ is ensured to have the integral factor and the steady-state errors for all these three cases from the limits in (11) to (13) are zero. This is the end of the proof.

For the step reference input excitation in Proposition 2, it is a classical study covering in nearly all control engineering textbooks. To extend such fundamental idea in a similar way, the steady-state errors due to other inputs, such as ramp or parabolic signals, for all these three excitations can also be formulated easily. In addition, Proposition 2 also provides a supportive reason for the popularity of PI and PID controllers. Since these two controllers have a form of (8) but just with improper transfer function $C_{1}(s)$, PI and PID controllers can therefore ensure zero steady-state errors for these three step excitations. However, their transfer functions do not agree with Proposition 1 so the sudden jump in the command signal is expected.

\section{Reference Model Matching}

Proposition 3: If $C_{1}(s)$ in the form of (14), the structure of $C(s)$ will have the integral factor as in (8).

$$
C_{1}(s)=\frac{s T(s)}{P(s)(1-T(s))},
$$

where

$$
T(s)=\frac{b_{m} s^{m}+b_{m-1} s^{m-1}+\ldots+b_{1} s+b_{0}}{s^{n}+a_{n-1} s^{n-1}+\ldots+a_{1} s+a_{0}}
$$


is a stable, proper $(n>m)$ and minimum-phase desired closed-loop transfer function from $R(s)$ to $Y(s)$ with the relative degree of $T(s)>$ the relative degree of $P(s)$, and at least (i) $b_{0}=a_{0}$ for a process without an integral factor and (ii) $b_{0}=a_{0}$ and $b_{1}=a_{1}$ for a process with an integral factor.

Proof: From (3), the closed-loop transfer function from $R(s)$ to $Y(s)$ is $G_{31}(s)$. Since $T(s)$ is strictly proper $(n>m), T(s) \neq 1$. By direct substitution, the closed-loop transfer function (16) is $T(s)$ as specified.

$$
\begin{aligned}
\frac{Y(s)}{R(s)} & =G_{31}(s)=\frac{P(s) C(s)}{1+P(s) C(s)}=\frac{P(s)\left(\frac{1}{s} C_{1}(s)\right)}{1+P(s)\left(\frac{1}{s} C_{1}(s)\right)} \\
& =\frac{\left(\frac{T(s)}{(1-T(s))}\right)}{1+\left(\frac{T(s)}{(1-T(s))}\right)}=\frac{T(s)}{(1-T(s))+T(s)}=T(s)
\end{aligned}
$$

If $P(s)$ has no integral factor so $a p_{0} \neq 0$ and let $T(s)$ be with $b_{0}=a_{0}$, then the transfer function $C_{1}(s)$ becomes (17).

$$
\begin{aligned}
C_{1}(s) & =\frac{s\left(\frac{b_{m} s^{m}+b_{m-1} s^{m-1}+\ldots+b_{1} s+a_{0}}{s^{n}+a_{n-1} s^{n-1}+\ldots+a_{1} s+a_{0}}\right)}{P(s)\left(1-\frac{b_{m} s^{m}+b_{m-1} s^{m-1}+\ldots+b_{1} s+a_{0}}{s^{n}+a_{n-1} s^{n-1}+\ldots+a_{1} s+a_{0}}\right)} \\
= & \frac{\left(b_{m} s^{m}+b_{m-1} s^{m-1}+\ldots+b_{1} s+a_{0}\right)}{P(s)\left(\left(s^{n-1}+a_{n-1} s^{n-2}+\ldots+a_{1}\right)-\left(b_{m} s^{m-1}+b_{m-1} s^{m-2}+\ldots+b_{1}\right)\right)}
\end{aligned}
$$

As a result, $\lim _{s \rightarrow 0} C_{1}(s) \neq 0$ or $C_{1}(s)$ has no zero at the origin. Therefore, $C(s)$ in $(8)$ has the integral factor as desired.

If $P(s)$ has an integral factor so $a p_{0}=0$ but $a p_{1} \neq 0$ and let $T(s)$ be with $b_{0}=a_{0}$ and $b_{1}=a_{1}$, then the transfer function $C_{1}(s)$ becomes (18).

$$
\begin{aligned}
C_{1}(s) & =\frac{s\left(\frac{b_{m} s^{m}+b_{m-1} s^{m-1}+\ldots+a_{1} s+a_{0}}{s^{n}+a_{n-1} s^{n-1}+\ldots+a_{1} s+a_{0}}\right)}{P(s)\left(1-\frac{b_{m} s^{m}+b_{m-1} s^{m-1}+\ldots+a_{1} s+a_{0}}{s^{n}+a_{n-1} s^{n-1}+\ldots+a_{1} s+a_{0}}\right)} \\
= & \frac{\left(b_{m} s^{m}+b_{m-1} s^{m-1}+\ldots+a_{1} s+a_{0}\right)}{P(s)\left(\left(s^{n-1}+a_{n-1} s^{n-2}+\ldots+a_{2} s\right)-\left(b_{m} s^{m-1}+b_{m-1} s^{m-2}+\ldots+b_{2} s\right)\right)}
\end{aligned}
$$


As a result, the integral factor in $P(s)$ is cancelled out and $\lim _{s \rightarrow 0} C_{1}(s) \neq 0$ or $C_{1}(s)$ has no zero at the origin. Therefore, $C(s)$ in (8) has the integral factor as desired.

From (17) and (18), since $T(s)$ is strictly proper, the relative degree of $C_{1}(s)$ is equal to relative degree of $T(s)-$ relative degree of $P(s)-1$. Therefore, if the relative degree of $T(s)>$ relative degree of $P(s)$, then the transfer function $C_{1}(s)$ is proper as desired. This is the end of the proof.

More precise extended outcomes of Proposition 3 are as follows. (i) If the relative degree of $T(s)$ is less than or equal to the relative degree of $P(s), C_{1}(s)$ is improper. (ii) If the relative degree of $T(s)$ is equal to $1+$ relative degree of $P(s), C_{1}(s)$ is proper but not strictly proper. (iii) If the relative degree of $T(s)$ is greater than $1+$ relative degree of $P(s), C_{1}(s)$ is strictly proper.

An inheriting scheme employing in Proposition 3 is to use the cancellation of the process model by the controller and placing the closed-loop model $T(s)$, which is at least strictly proper and stable, as desired. The selection of $T(s)$ can be conducted from various algorithms -for instance, pole-placement. The main requirement is just its structure and its coefficients of the lower order term(s). In addition, since this paper considers only the process $P(s)$ that is minimum-phase, proper, without zero at the origin, and with all LHP pole(s) without or with an integral factor, there is no unstable pole-zero cancellation between the process model and controller. The closed-loop system in Figure 1 is therefore internally stable. However, the sensitivity and robustness of the control system must be considered in practical usage but not included in the scope of this paper.

\section{Generalized Integral Anti-Windup}

In case of the system suffering actuator saturation, anti-windup scheme has long been in use especially for PI and PID controllers. In this paper, the extension of the anti-windup scheme for the integral action portion of all SISO controllers with an integral factor including the presented one in (8) is provided based on the back calculation method.

For the controller $C(s)$ in (2) in the form in (8) with $C_{1}(s)$ in (9), $a c_{0}=0$ and it can be written in the form of (19).

$$
\begin{aligned}
C(s) & =\frac{b c_{m c} s^{m c}+b c_{m c-1} s^{m c-1}+\ldots+b c_{1} s+b c_{0}}{s^{n c}+a c_{n c-1} s^{n c-1}+\ldots+a c_{1} s} \\
& =\frac{q_{M} s^{M}+q_{M-1} s^{M-1}+\ldots+q_{1} s+q_{0}}{s\left(s^{N}+r_{N-1} s^{N-1}+\ldots+r_{1} s+r_{0}\right)}
\end{aligned}
$$

The controller can then be expressed in a parallel form (20) where $k_{i}$ and $\bar{C}(s)$ are given in (21) and (22) respectively when $\bar{q}_{0}=q_{1}-r_{1} k_{i}, \ldots, \bar{q}_{M-1}=q_{M}-r_{M} k_{i}$.

$$
\begin{gathered}
C(s)=\frac{k_{i}}{s}+\bar{C}(s) \\
k_{i}=\frac{q_{0}}{r_{0}}
\end{gathered}
$$




$$
\bar{C}(s)=\frac{\bar{q}_{M-1} s^{M-1}+\bar{q}_{M-1} s^{M-2}+\ldots+\bar{q}_{1} s+\bar{q}_{0}}{s^{N}+r_{N-1} s^{N-1}+\ldots+r_{1} s+r_{0}}
$$

The control system with the generalized integral anti-windup for the controller (20) based on the back calculation method can then be implemented as in Figure 2. Denote that $k_{a w}$ is an anti-windup gain that can be adjusted freely. In general, $k_{a w}$ should be large enough to stop the integrator when the system suffers actuator saturation. One common selection is to set $k_{a w}=k_{i}[14]$.

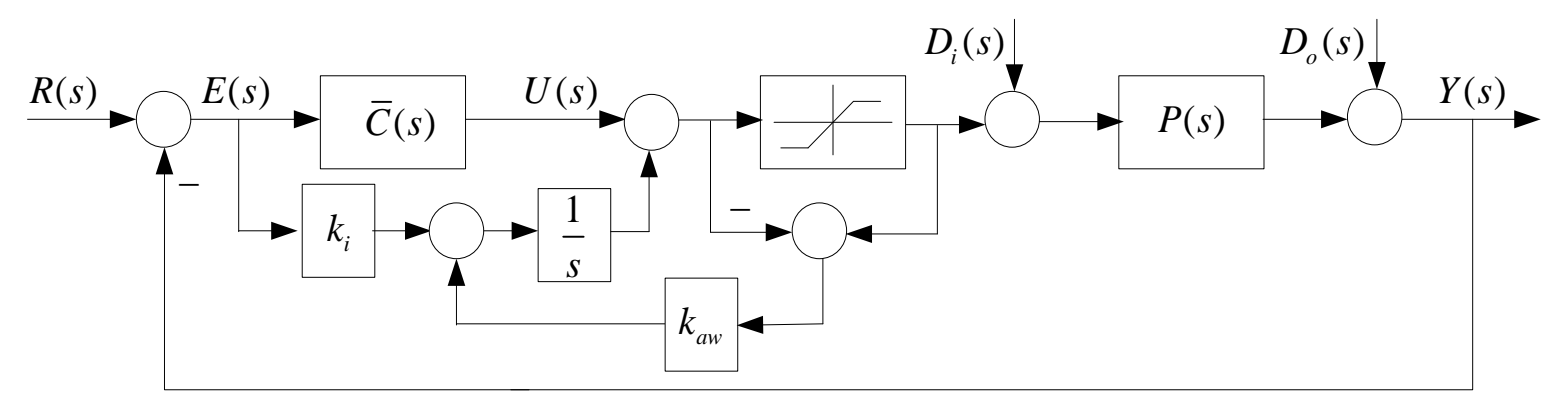

Figure 2. Control system with a generalized integral anti-windup

Note that the proposed scheme is added the anti-windup action to only the integral factor which can be considered as the extension of the well-known conventional PI/PID anti-windup scheme to avoid the difficulty in design and implementation comparing to other anti-windup compensators. Since the remaining action in $\bar{C}(s)$ is faster than the integral action, the windup effect, if any, is lesser and the improvement in employing this generalized integral anti-windup could be expected.

The implementation of the proposed controller $C(s)$ either in the form of (8) or (20) can be directly extended the realization of real rational proper transfer function which is well-established and readily available in most signals and systems textbooks. One of such examples is the implementation by analogue circuits [19].

\section{Simulation Results}

In this section, some numerical examples in designing the controller based on the proposed structure with and without generalized integral anti-windup are demonstrated. The comparisons are mainly with a PID controller in the form of (22) where $K_{P}$ is a proportional gain, $K_{I}$ is an integral gain, $K_{D}$ is a derivative gain and $N_{F}$ is a filter coefficient for derivative control. In case of ideal PID controller, $N_{F}$ approaches infinity.

$$
C_{P I D}(s)=K_{P}+\frac{K_{I}}{s}+\frac{K_{D} s}{\frac{1}{N_{F}} s+1}
$$

\section{Case Study 1: Process without Integral Factor}

An example process model of the pitch control of wind turbine in [20] as rationally expanded in (24) is considered. In this case, the relative degree of $P(s)$ is 2 and it has no integral factor. 


$$
P(s)=\frac{2.7027}{s^{2}+6.0541 s+8.1081}
$$

The PID controller's gains for the (Fuzzy) PID controller in [20] are $K_{P}=0.5$, $K_{I}=0.75$ and $K_{D}=0$ (and $N_{F}$ can be any non-zero number) which is just a PI controller. The resulting closed-loop transfer function $G_{31}(s)$ is as $T_{P I}(s)$ in (25) with the relative degree of 2 .

$$
T_{P I}(s)=\frac{1.3514 s+2.0270}{s^{3}+6.0541 s^{2}+9.4595 s+2.0270}
$$

This $T_{P I}(s)$ may be selected as the desired closed-loop transfer function $T(s)$ for the proposed controller design, but the resulting controller is just the same PI controller which is not strictly proper. According to Proposition 3, $T(s)$ must have the relative degree greater than that of the process model, in this case is 2 , so at least the relative degree of $T(s)$ must be 3 or more. For direct comparison, a factor with one extra non-dominant pole at $s=-5$ with unity DC gain is added to $T_{P I}(s)$ in (25) so the choice of $T(s)$ becomes the one in (26). Note that the coefficients of the zero-order term in the numerator and denominator are equal as required.

$$
T(s)=\left(\frac{1.3514 s+2.0270}{s^{3}+6.0541 s^{2}+9.4595 s+2.0270}\right)\left(\frac{5}{s+5}\right)
$$

The resulting controller can be derived based on (8) and (14), which is strictly proper. It can be expressed in both forms of (8) and (20) as shown in (27).

$$
\begin{aligned}
C(s) & =\frac{1}{s}\left(\frac{2.5000 s^{3}+18.8851 s^{2}+42.9730 s+30.4054}{s^{3}+11.0541 s^{2}+39.7297 s+42.5676}\right) \\
& =\frac{0.7143}{s}+\frac{1.7857 s^{2}+10.9894 s+14.5946}{s^{3}+11.0541 s^{2}+39.7297 s+42.5676}
\end{aligned}
$$

The simulation results due to unit step reference input at $t=0 \mathrm{~s},-0.5$ step output disturbance entering at $t=25 \mathrm{~s}$, and -0.5 step input disturbance entering at $t=50 \mathrm{~s}$ using both controllers are shown in Figure 3. As can be seen from Figure 3(a), since both controllers having an integral factor as pointed in Proposition 2, both can track the step reference input and eliminate the effect of step input and output disturbances. In this case, the responses of both PI and proposed controller are closed to each other because both yield similar closed-loop models. Since the non-dominant pole is added in the desired closed-loop model for designing the proposed controller, its response is slightly slower as expected. However, when observing the corresponding command signals in Figure 3(b), the inheriting advantage of the proposed controller can be verified. There are no sudden jump or surge in its command signal of the proposed controller while the PI controller causes sudden jumps under step reference input excitation (about 0.5 unit) and step output disturbance excitation (about 0.25 unit). Denote that the sudden jumps are not clearly seen due to very slow response design. If the nonlinear rate limiter is included in the actuator, then the system with the proposed controller should be less suffered. 


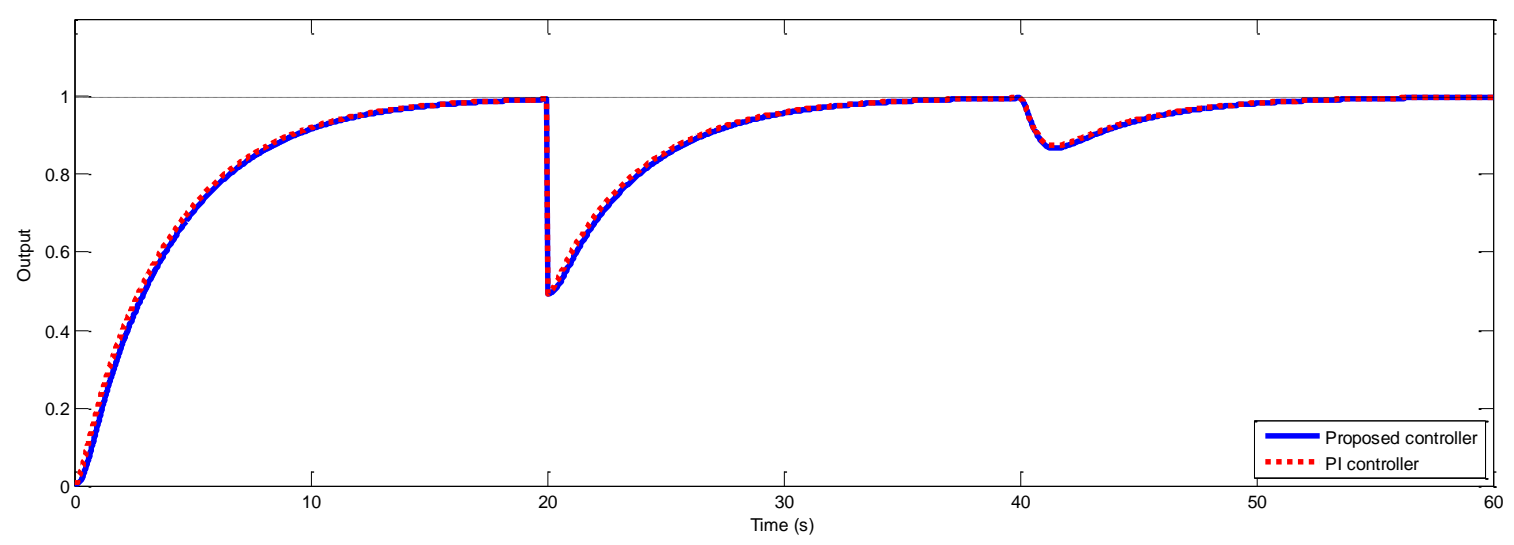

(a) Output

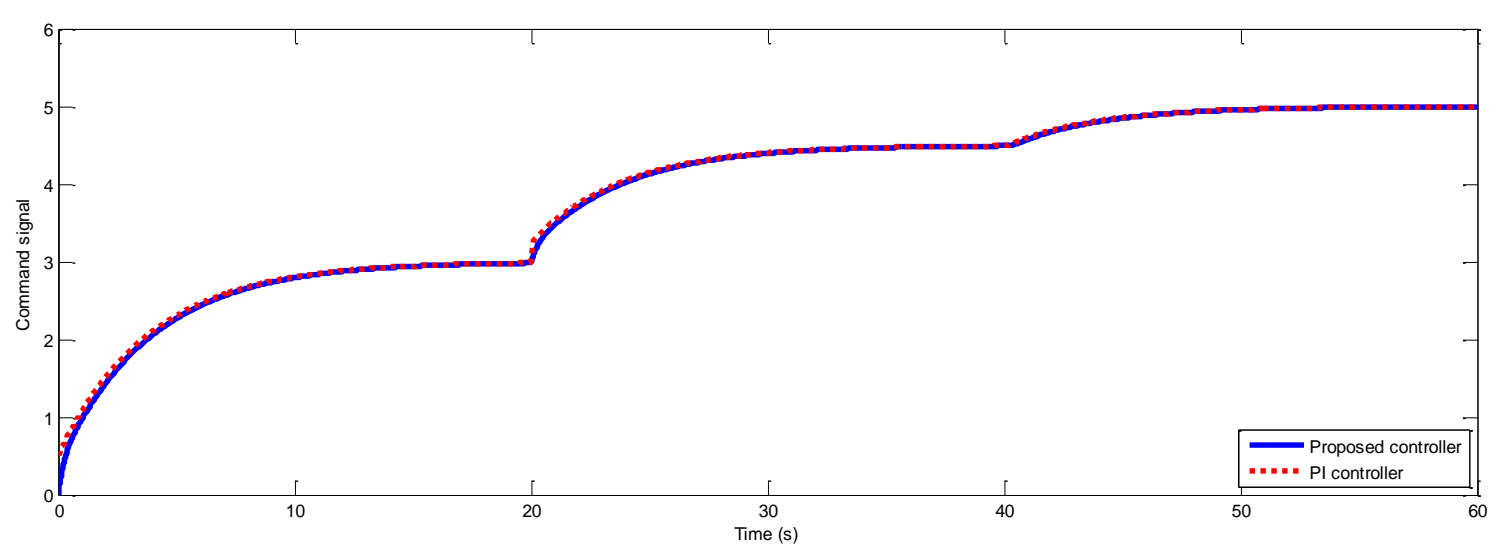

(b) Command signal

Figure 3. Simulation results of case study 1

Since the above PI controller, and hence consequently the proposed controller, still causes a slow response, the automatic tuning by MATLAB is used to tune the PID controller's gains. The results are $K_{P}=5.4209, K_{I}=10.1496$ and $K_{D}=0.1417$ with $N_{F}=10.3842$. Following the same concept, the closed-loop transfer function of PID control system with the extra non-dominant pole at $s=-15$ is used as the desired closed-loop model for the proposed controller design. The resulting controller is expressed in (28) and the simulation results due to unit step reference input at $t=0 \mathrm{~s},-0.5 \mathrm{step}$ output disturbance entering at $t=5 \mathrm{~s}$, and -0.5 step input disturbance entering at $t=10 \mathrm{~s}$ using both controllers are depicted in Figure 4.

$$
\begin{aligned}
C(s) & =\frac{1}{s}\left(\frac{103.38 s^{4}+1622.5 s^{3}+8452.8 s^{2}+17653 s+12818}{s^{4}+31.438 s^{3}+336.18 s^{2}+1328.4 s+1547.8}\right) \\
& =\frac{8.2817}{s}+\frac{95.102 s^{3}+1362.2 s^{2}+5668.7 s+6650.5}{s^{4}+31.438 s^{3}+336.18 s^{2}+1328.4 s+1547.8}
\end{aligned}
$$

From Figure 4(b), it can be clearly seen the advantage of using the proposed controller so that the sudden jump in command signal can be avoided comparing to the command signal of PID control system which exhibits large jumps clearly. In addition, if the ideal derivative action in PID controller is used without the filter, it would cause an impulsive surge in its command signal due to step reference input and step output disturbance excitations as will be demonstrated in Case Study 2. 


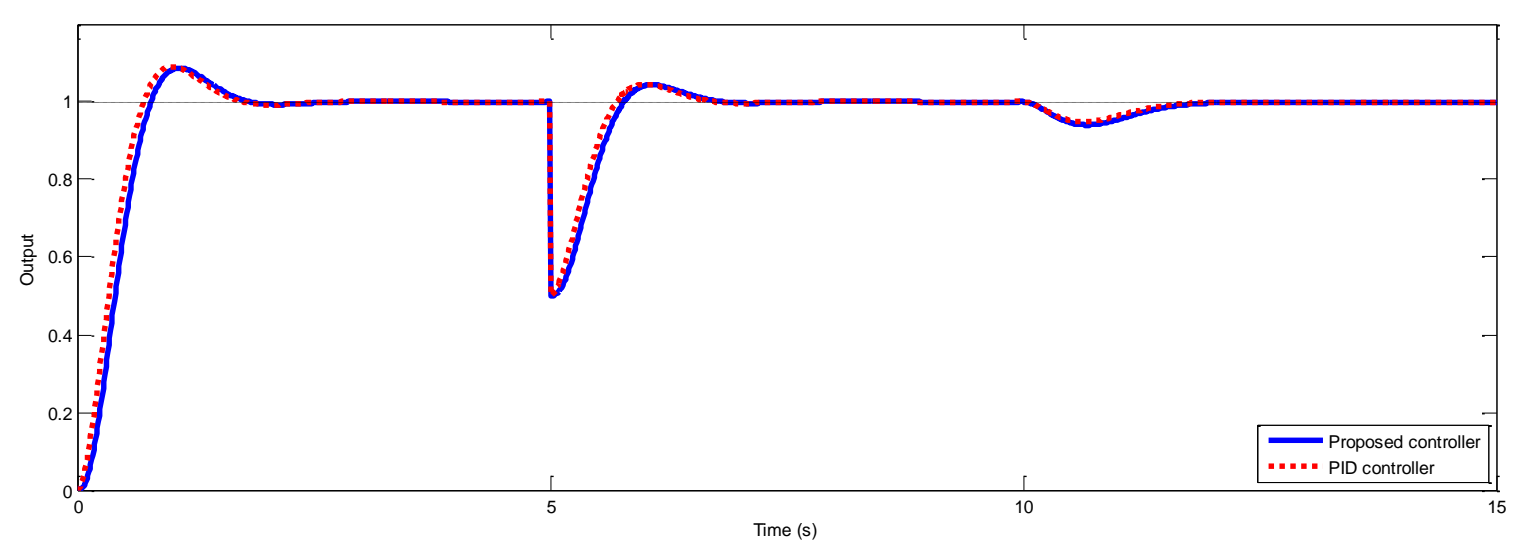

(a) Output

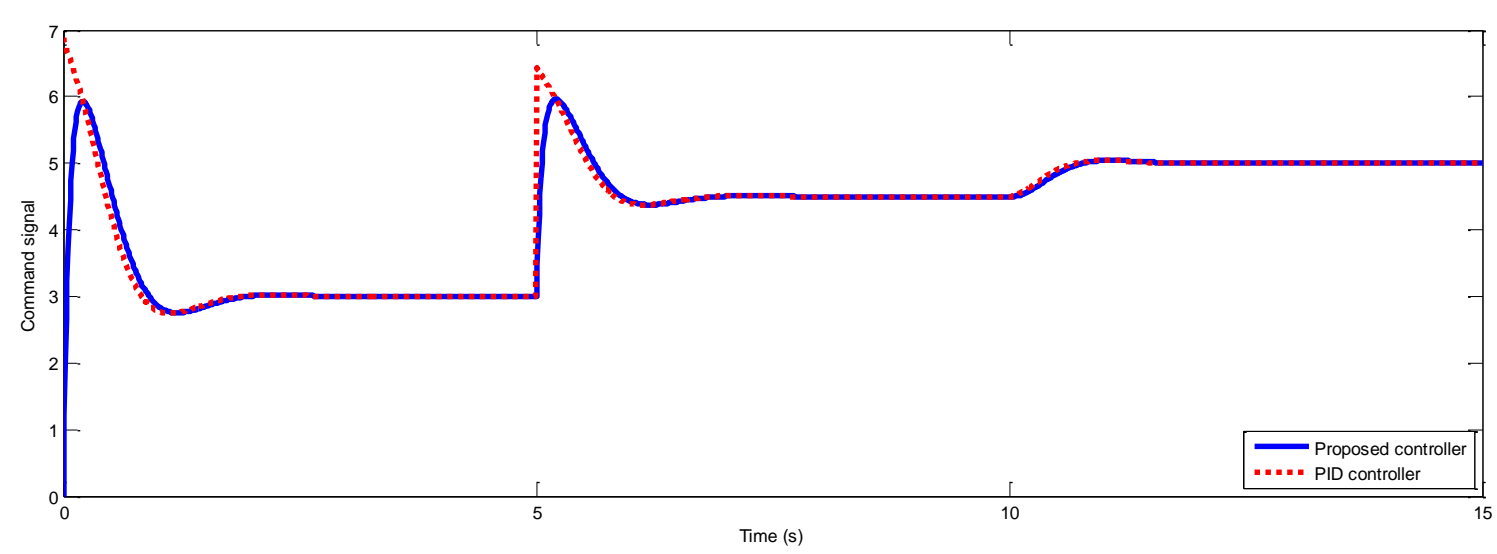

(b) Command signal

Figure 4. Simulation results of case study 1 after tuning

\section{Case Study 2: Process with Integral Factor}

In this case study, the position control of sun tracker panel in [21] is investigated with the numerically summarized transfer function in (24). Notice that $P(s)$ has an integral factor and its relative degree is 3 .

$$
P(s)=\frac{140625}{s\left(s^{2}+6251 s+146875\right)}
$$

For comparison, the ideal CSA PID controller in [21] with $K_{P}=9.99999$, $K_{I}=8.11378$ and $K_{D}=0.00010$ is considered. In this case, of the system using the given PID controller is firstly observed for the selection of $T(s)$ based on poles, zeroes and its step response. Note that the relative degree of $P(s)$ is 3 , the relative degree of $T(s)$ must be at least 4 . And since $P(s)$ has a pole at the origin, $T(s)$ must have at least one zero to allow the numerator having two terms of which its coefficients must be equal to the two lowest coefficients of the denominator. Thus, the possible lowest order $T(s)$ is 5. From these observations, the desired closed-loop transfer function $T(s)$ is arbitrarily selected by placing the closed-loop poles, that should yields nearly the same response speed as the PID 
control system, at $s=-6,-6,-6,-200$ and -500 and let the numerator meet the required condition by setting $b_{0}=a_{0}$ and $b_{1}=a_{1}$. The resulting $T(s)$ is given in (30).

$$
T(s)=\frac{5497200 s+10800000}{s^{5}+468 s^{4}+58208 s^{3}+948816 s^{2}+5497200 s+10800000}
$$

Then following the design same design steps, the controller in (31) is obtained.

$$
\begin{aligned}
& C(s)=\frac{1}{s}\left(\frac{39.0912 s^{3}+2.44436 \times 10^{5} s^{2}+6.22160 \times 10^{6} s+1.12800 \times 10^{7}}{s^{3}+468 s^{2}+58208 s+9.48816 \times 10^{5}}\right) \\
& =\frac{11.8885}{s}+\frac{27.2027 s^{2}+2.38872 \times 10^{5} s+5.52959 \times 10^{6}}{s^{3}+468 s^{2}+58208 s+9.48816 \times 10^{5}}
\end{aligned}
$$

For the simulations, the unit step reference input at $t=0 \mathrm{~s},-0.5$ step output disturbance entering at $t=2 \mathrm{~s}$, and -1 step input disturbance entering at $t=4 \mathrm{~s}$ are applied to both control systems. The results are shown in Figure 5.

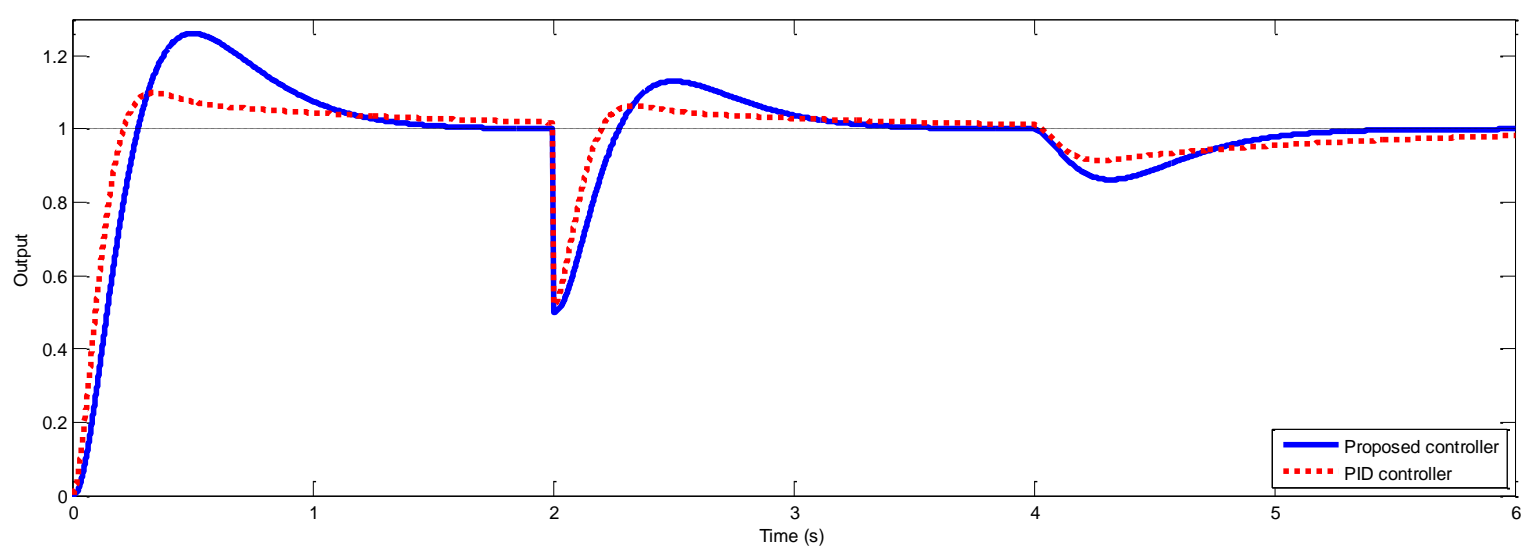

(a) Output

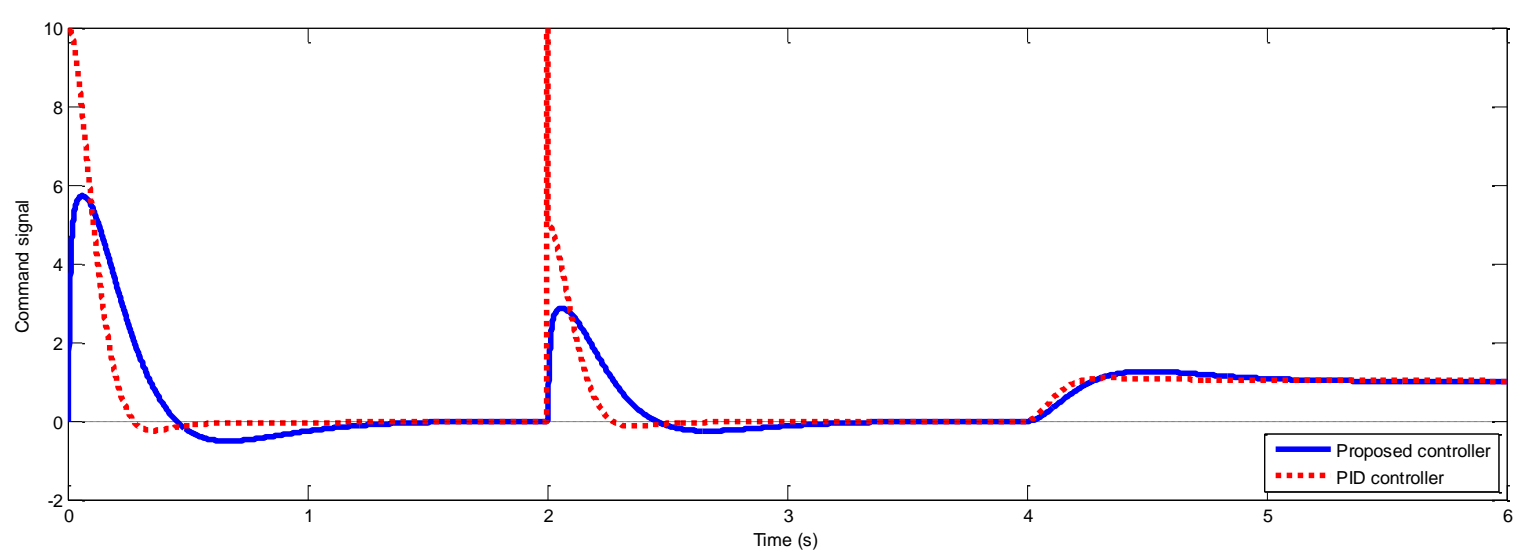

(b) Command signal

Figure 5. Simulation results of case study 2 
From Figure 5, it should be noted the capability of the proposed controller in tracking the step reference input and eliminating the effect of step input and output disturbances while avoiding sudden jumps in the command signal due to these excitations. However, due to the numerator placing constraint of $T(s)$, the proposed control system yields about $25 \%$ overshoot; although, all real closed-loop poles are selected in $T(s)$.

\section{Case Study 3: Generalized Integral Anti-windup}

In this part, the saturation effect in the command signal is investigated. The proposed control system designed in Case Study 2 is directly employed. From Figure 5(b), the PID control system would suffer from the actuator saturation more or less at any level of saturation due to impulsive surge from the derivative action but would be more affected if the saturation level is below 10 units. To lessen the windup effect, the anti-windup control for PID controller has long been proposed and in used. The simulations for the PID control system are therefore excluded in this case study. However, for the proposed control system, the maximum command signal due to these excitations is around 5.7237 units so it would also suffer from the actuator saturation at any level below this value. This case study, therefore, demonstrates the effectiveness of the generalized integral anti-windup scheme proposed in this paper.

The simulations of the control systems with the actuator saturation at \pm 4 units and \pm 2 units under the step reference input and output disturbance excitations are conducted when the anti-windup gain is set as $k_{a w}=k_{i}=11.8885$. The comparing results for the system with and without generalized integral anti-windup are shown in Figure 6 and Figure 7 for the saturation levels at at \pm 4 units and \pm 2 units respectively.

It is clear from the results in Figure 6 and Figure 7 that the lower the level of saturation, the more the effect to the response. However, once the proposed generalized integral anti-windup is utilized, the saturation in the command signal is shorter and the effect to the output is significantly less. The saturation effect is distinctively alleviated.

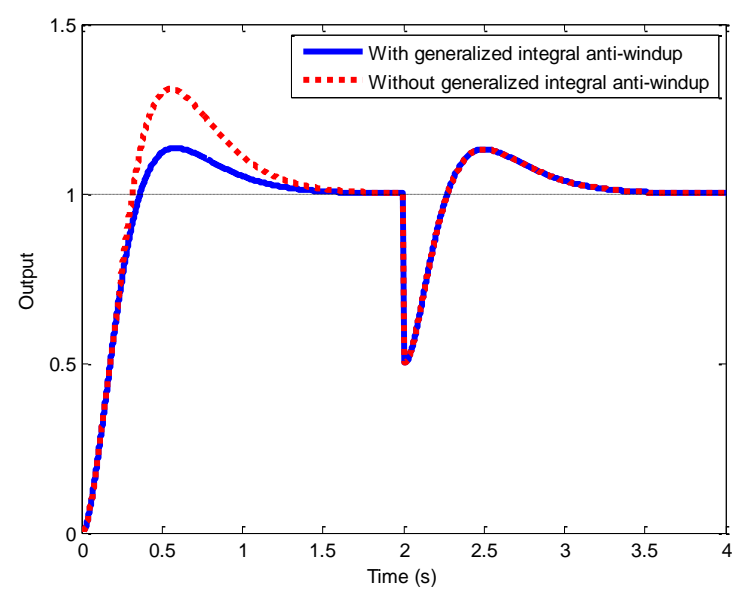

(a) Output

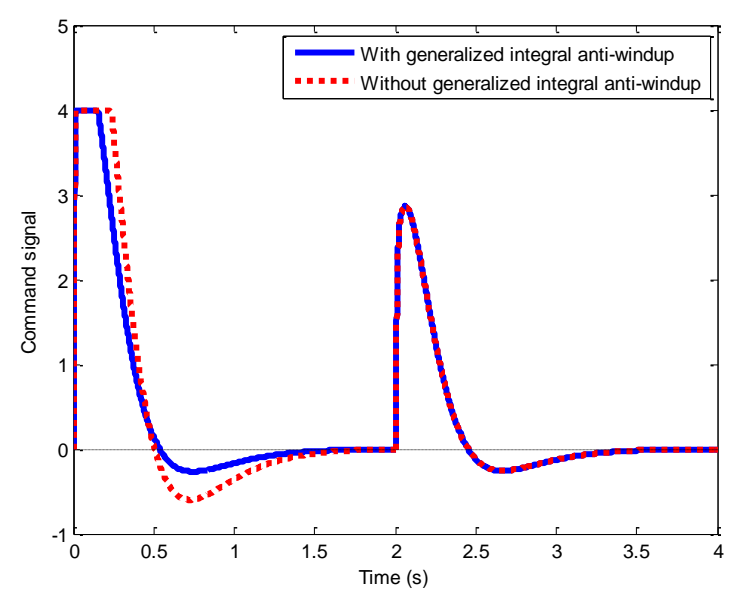

(b) Command signal

Figure 6. Simulation results of case study 3 under the actuator saturation at \pm 4 units 


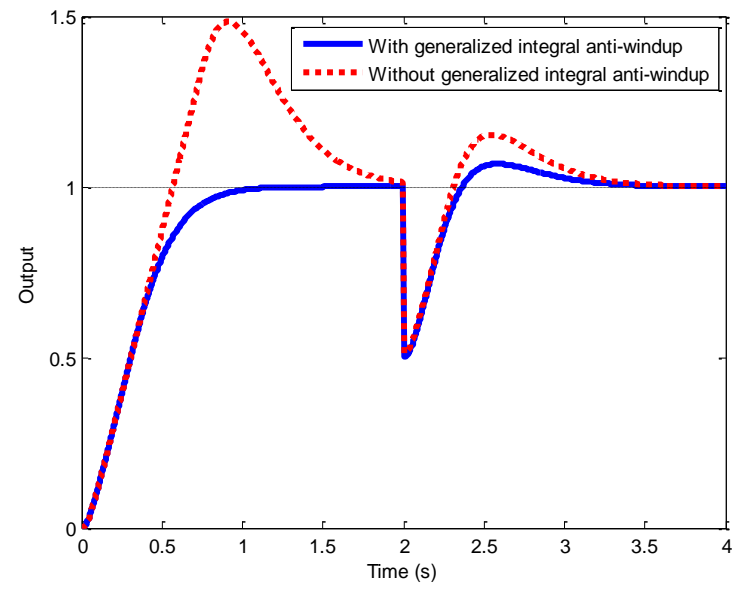

(a) Output

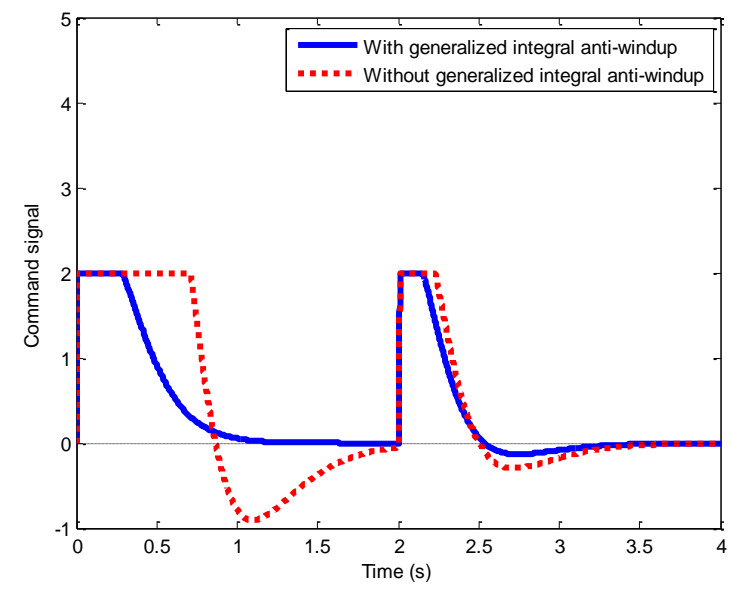

(b) Command signal

Figure 7. Simulation results of case study 3 under the actuator saturation at \pm 2 units

\section{Conclusions}

This paper introduces the structure of a controller to assure two basic requirements. First, the controller should be strictly proper to avoid sudden jump in the command signal due to step reference input excitation and step input and output disturbances excitations. Second, the controller must, at least, have an integral factor to eliminate the steady-state error due to the same step excitations. Such controller can be simply designed based on reference model matching and model cancellation with only two required conditions on the desired closed-loop transfer function which are its relative degree and the equality of the lower order coefficient(s) in its numerator and denominator. In addition, the paper proposes the generalized integral anti-windup structure to handle the windup effect due to the integral factor of the controller when the system is subjected to the actuator saturation. The simulation results verify the success in using these proposed concepts to achieve all mentioned targets.

\section{References}

[1] P. Shipley, "A unified approach to synthesis of linear systems," IEEE Transactions on Automatic Control, Vol. 8, No. 2, pp. 114-125, 1963.

[2] C.T. Chen, "Introduction to the linear algebraic method for control system design," IEEE Control Systems Magazine, Vol. 7, No. 5, pp. 36-42, 1987.

[3] D.C. Youla, H.A. Jabri, and J.J. Bongiorno, "Modern Wiener-Hopf design of optimal controllers--Part II: The multivariable case," IEEE Transactions on Automatic Control, Vol. 21, No. 3, pp. 319-338, 1976.

[4] V. Kučera, "Stability of discrete linear feedback systems," In: 6th IFAC World Congress (IFAC 1975) - Part 1: Theory, Boston/Cambridge, Massachusetts, United States, pp. 573-578, 1975.

[5] B.C. Moore, and L.M. Silverman, "Model matching by state feedback and dynamic compensation," IEEE Transactions on Automatic Control, Vol. 17, No. 4, pp. 491-497, 1972.

[6] Ş. Sabău, and N.C. Martins, "Youla-like parametrizations subject to qi subspace constraints," IEEE Transactions on Automatic Control, Vol. 59, No. 6, pp. 1411-1422, 2014. 
[7] Y.S. Wang, N. Matni, and J.C. Doyle, "A system-level approach to controller synthesis" IEEE Transactions on Automatic Control, Vol. 64, No. 10, pp. 4079-4093, 2019.

[8] H. Sun, T. Asai, R. Ariizumi, and S. Azuma, "Model matching for mimo reference models with time varying parameter," In: 12th Asian Control Conference (ASCC), IEEE, Kitakyushu-shi, Japan, 2019.

[9] G. Ziegler, and N.B. Nichols, "Optimum setting for automatic controllers," Transactions of ASME, Vol. 64, No. 11, pp. 759-768, 1942.

[10] N.H. Nguyen, and P.D. Nguyen, "Overshoot and settling time assignment with PID for first-order and second-order systems," IET Control Theory and Applications, Vol. 12, No. 17, pp. 2407-2416, 2018.

[11] S. Somasundaram, and T. Benjanarasuth, "CDM based two degree of freedom PI controller tuning rules for stable and unstable FOPTD processes and pure integrating processes with time delay," International Journal of Automation and Control, Vol. 13 No. 3, pp. 263-281, 2019. doi: 10.1504/ijaac.2019.10019360

[12] H.A. Fertik, and C.W. Ross, "Direct digital control algorithms with anti-windup feature," ISA Transactions, Vol. 6, No. 4, pp. 317-328, 1967.

[13] J.C. Doyle, R.S. Smith, and D.F. Enns, "Control of plants with input saturation nonlinearities," In: 1987 American Control Conference, Minneapolis, Minnesota, United States, pp. 1034-1039, 1987.

[14] K.J. Åström, and L. Rundqwist, "Integrator windup and how to avoid it," In: 1989 American Control Conference, Pittsburgh, Pennsylvania, United States, pp. 1693-1698, 1989.

[15] H. Jigang, W. Jie, and F. Hui, "An anti-windup self-tuning fuzzy PID controller for speed control of brushless DC motor," Automatika, Vol. 58, No. 3, pp. 321-335, 2017.

[16] L.R. Silva, R.C.C. Flesch, and J.E. Normey-Rico, "Analysis of anti-windup techniques in PID control of processes with measurement noise," In: The 3rd IFAC Conference on Advances in Proportional-Integral-Derivative Control, IFAC, Ghent, Belgium, 2018. doi: 10.1016/j.ifacol.2018.06.100

[17] S. Al-Haddad, and H. Wahid, "Decoupled integral LQR controller with anti-windup compensator for MIMO two rotor aerodynamical system (tras)," Journal of Engineering Science and Technology, Vol. 14, No. 3, pp. 1374-1397, 2019 [Online]. Available: http://jestec.taylors.edu.my/Vol\%2014\%20issue\%203\%20June\%202019/14_3_20.pdf

[18] A.J. Veronica, and N.S. Kumar, "Load frequency controller design for microgrid using internal model control approach," International Journal of Renewable Energy Research, Vol. 7, No. 2, pp. 778-786, 2017 [Online]. Available: https://www.ijrer.org/ijrer/index.php/ijrer/article/download/5655/pdf

[19] C.T. Chen, Signals and Systems, $3^{\text {rd }}$ Edition, Oxford University Press, New York, New York, United States, 2004.

[20] S. Baburajan, and A. Ismail, "Pitch control of wind turbine through PID, fuzzy and an adaptive fuzzy-PID controller," International Research Journal of Engineering and Technology, Vol. 4, No. 9, pp. 669-675, 2017 [Online]. Available: https://www.irjet.net/archives/V4/i9/IRJET-V4I9115.pdf

[21] S.D. Hanwate, and Y.V. Hote, "Design of PID controller for sun tracker system using QRAWCP approach," International Journal of Computational Intelligence Systems, Vol. 11, pp. 133-145, 2018. doi: 10.2991/ijcis.11.1.11 